\title{
Hybrid, metal oxide-peptide amphiphile micelles for molecular magnetic resonance imaging of atherosclerosis
}

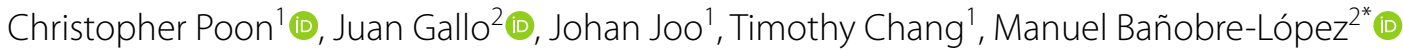
and Eun Ji Chung 1,3,4,5,6*

\begin{abstract}
Background: Atherosclerosis, a major source of cardiovascular disease, is asymptomatic for decades until the activation of thrombosis and the rupture of enlarged plaques, resulting in acute coronary syndromes and sudden cardiac arrest. Magnetic resonance imaging (MRI) is a noninvasive nuclear imaging technique to assess the degree of atherosclerotic plaque with high spatial resolution and excellent soft tissue contrast. However, MRI lacks sensitivity for preventive medicine, which limits the ability to observe the onset of vulnerable plaques. In this study, we engineered hybrid metal oxide-peptide amphiphile micelles (HMO-Ms) that combine an inorganic, magnetic iron oxide or manganese oxide inner core with organic, fibrin-targeting peptide amphiphiles, consisting of the sequence CREKA, for potential MRI imaging of thrombosis on atherosclerotic plaques.

Results: Hybrid metal oxide-peptide amphiphile micelles, consisting of an iron oxide (Fe-Ms) or manganese oxide (Mn-Ms) core with CREKA peptides, were self-assembled into 20-30 nm spherical nanoparticles, as confirmed by dynamic light scattering and transmission electron microscopy. These hybrid nanoparticles were found to be biocompatible with human aortic endothelial cells in vitro, and HMO-Ms bound to human clots three to five times more efficiently than its non-targeted counterparts. Relaxivity studies showed ultra-high $r_{2}$ value of $457 \mathrm{mM}^{-1} \mathrm{~s}^{-1}$ and $r_{1}$ value of $0.48 \mathrm{mM}^{-1} \mathrm{~s}^{-1}$ for Fe-Ms and $\mathrm{Mn}-\mathrm{Ms}$, respectively. In vitro, MR imaging studies demonstrated the targeting capability of CREKA-functionalized hybrid nanoparticles with twofold enhancement of MR signals.
\end{abstract}

Conclusion: This novel hybrid class of MR agents has potential as a non-invasive imaging method that specifically detects thrombosis during the pathogenesis of atherosclerosis.

Keywords: Hybrid nanoparticle, Micelle, Targeting, Magnetic resonance imaging, Atherosclerosis, Iron oxide, Manganese oxide

\footnotetext{
*Correspondence: manuel.banobre@inl.int; eunchung@usc.edu ${ }^{1}$ Department of Biomedical Engineering, University of Southern California, 1042 Downey Way, Los Angeles, CA 90089, USA

${ }^{2}$ Advanced (Magnetic) Theranostic Nanostructures Lab, Department of Life Sciences, International Iberian Nanotechnology Laboratory, Avenida Mestre José Veiga, Braga, Portugal

Full list of author information is available at the end of the article
} 


\section{Background}

Cardiovascular disease (CVD) is the leading cause of death in the United States, with over 800,000 deaths each year [1]. Atherosclerosis, which represents close to $70 \%$ of all CVDs, is a degenerative inflammatory disease that leads to the formation of thrombosis and increased build-up of rupture-prone or vulnerable plaques [2]. Its progression is often asymptomatic for decades until the onset of acute cardiovascular events, such as myocardial infarction or stroke, thereby making early detection of atherosclerosis difficult $[3,4]$. Noninvasive nuclear imaging techniques, such as magnetic resonance imaging (MRI), have recently been used to diagnose coronary artery disease and preliminarily characterize plaque structure [5]. MRI has high spatial resolution and excellent soft tissue contrast, and does not require the use of ionizing radiation, making repetitive morphological follow-up possible [6, 7]. Nevertheless, MRI has limited sensitivity, making it difficult to differentiate and target diseased sites with conventional contrast agents.

Nanocarriers offer an alternative to conventional contrast agents and have the ability to carry a high content of contrast agents and target diseased areas due to the enhanced permeability and retention (EPR) effect characteristic to leaky endothelium and damaged vasculature found in atherosclerotic plaques [8-17]. A variety of inorganic nanomaterials (e.g. quantum dots, gold, carbon nanotubes, mesoporous silica metal oxide) have been investigated for cardiovascular diseases [18-20]. Specifically, iron oxide (Fe-NPs) and manganese oxide nanoparticles (Mn-NPs) are effective MRI contrast agents that have been used to image a wide variety of diseases [21-23]. Fe-NPs are negative contrast agents that reduce the transverse relaxation time $\left(T_{2}\right)$ to produce dark contrast-enhanced signals, while Mn-NPs are paramagnetic agents that shorten the longitudinal relaxation time $\left(T_{1}\right)$ of water proton to increase the bright signal intensity of a bright signal [24, 25]. However, most of these inorganic nanoparticles face problems with cellular toxicity and nonbiodegradability $[18,26,27]$. To mediate these issues, inorganic nanomaterials can be functionalized with organic components to improve colloidal stability, biocompatibility, biodegradability, and enhanced magnetic properties for imaging [28-31].

Previously, we have incorporated the fibrin-binding peptide, CREKA, into a class of organic nanoparticles called peptide amphiphile micelles (PAMs) for targeting thrombosis in atherosclerosis [32-35]. The CREKA micelles bind to the entire surface of the plaque, but concentrate at the shoulder portion of the plaque, a location that is prone to rupture and consist of microthrombi [36]. To combine the beneficial features of both organic and inorganic nanoparticles, in this work, we develop hybrid metal oxide PAMs (HMO-Ms) that consist of a highly crystalline iron or manganese oxide core encapsulated with organic, fibrin-targeting peptide amphiphiles for potential MRI detection of vulnerable plaques. HMO-Ms were self-assembled using a dry-film hydration method and found to be monodisperse, spherical nanoparticles of $\sim 20-30 \mathrm{~nm}$ and stable in presence of serum. In vitro biocompatibility assays showed that these hybrid nanoparticles were nontoxic to human aortic endothelial cells (hAECs), and HMO-Ms demonstrated to have three to five times higher binding affinity than non-targeted PAMs on fibrin-containing clots derived from human plasma and thrombin. Moreover, the iron oxide- and manganese oxide-containing PAMs (Fe-Ms and Mn-Ms), exhibited enhanced $T_{2^{-}}$and $T_{1}$-MRI-contrast, respectively. Our results highlight the potential of these inorganic/organic hybrid nanoparticles as highly effective delivery vehicles for contrast agents in MRI applications.

\section{Results and discussion \\ Preparation and characterization of HMO-Ms}

Metal oxide nanoparticles made of $\mathrm{Fe}_{3} \mathrm{O}_{4}$ (magnetite) were prepared following thermal decomposition protocols from iron acetylacetonate [37]. Transmission electron microscopy (TEM) showed spherical morphology with a diameter of $3.7 \pm 0.8 \mathrm{~nm}$ (Additional file 1: Fig. S1A, B). Similarly, Mn-NP were also fabricated following thermal decomposition protocols using manganese oleate [38]. TEM confirmed Mn-NP were spherical in shape with a diameter of $11.1 \pm 1.1 \mathrm{~nm}$ (Additional file 1: Fig. S2A, B). Fe and Mn presence in Fe-NP and Mn-NP, respectively, was corroborated by EDXS (Additional file 1: Figs. S1C, S2C). In both cases, nanoparticles were coated with oleate, which provided a lipid monolayer for functionalization with peptide amphiphiles (PAs).

To synthesize PAs, the fibrin binding peptide, CREKA, was conjugated onto DSPE-PEG(2000)-maleimide via thioether linkage (Additional file 1: Fig. S3). Using dryfilm hydration methods, varying amount of Fe-NPs, from $0.05 \mu \mathrm{mol}$ to $0.5 \mu \mathrm{mol}$, were modified with $50 \mathrm{nmol}$ of DSPE-PEG(2000)-CREKA (100 $\mu \mathrm{M}$ PAMs) (Additional file 1: Table S1). No aggregation was observed by dynamic light scattering (DLS) for $0.05 \mu \mathrm{mol}$ and $0.25 \mu \mathrm{mol}$ Fe-NP, and diameters $(<20 \mathrm{~nm})$ for hybrid nanoparticles were similar to that of CREKA-Ms without any Fe-NP. For $0.5 \mu \mathrm{mol}$ Fe-NP samples, a small sediment was precipitated at the bottom of the solution, indicating $0.5 \mu \mathrm{mol} \mathrm{Fe}-\mathrm{NP}$ is above the maximum loading capacity for $100 \mu \mathrm{M}$ PAMs (Additional file 1: Fig. S4). Therefore, 5:1 molar ratio of Fe-NP and DSPE-PEG(2000)-CREKA was chosen as optimal and scaled up accordingly. Similar loading experiments were performed between 0.05 and $1 \mu \mathrm{mol} \mathrm{Mn-NP}$ for $100 \mu \mathrm{M}$ PAMs, and 10:1 mol ratio 
Mn-NP and DSPE-PEG(2000)-CREKA was found as the optimal working ratio (Additional file 1: Table S2).

To further enhance the stability and permeability of HMO-Ms, 1,2-dioleoyl-sn-glycero-3-phosphocholine (DOPC) and cholesterol were incorporated together with DSPE-PEG(2000)-CREKA onto the oleate exterior of the metal oxide core to form an asymmetric bilayer. DOPC is commonly used on the surface of nanoparticles to mimic the lipid bilayer of cells, which enhances the interaction with cells $[39,40]$. About $20-50 \mathrm{~mol} \%$ of cholesterol is found in the phospholipid bilayer of mammalian cells, which provides order and stability to the membrane structure $[41,42]$. In this study, $37 \mathrm{~mol} \%$ cholesterol was used in our formulation of HMO-Ms. Driven by hydrophobic interactions, Fe-NP (5 molar) and Mn-NP (10 molar) were coated with DOPC, cholesterol, and DSPEPEG(2000)-CREKA at 1.33:1.33:1 molar ratio (Fig. 1a). Encapsulation of Fe-NP and Mn-NP within the asymmetric bilayer yielded slightly larger particles than the Fe-NP and Mn-NP core, with diameters of $20.4 \pm 7.9 \mathrm{~nm}$

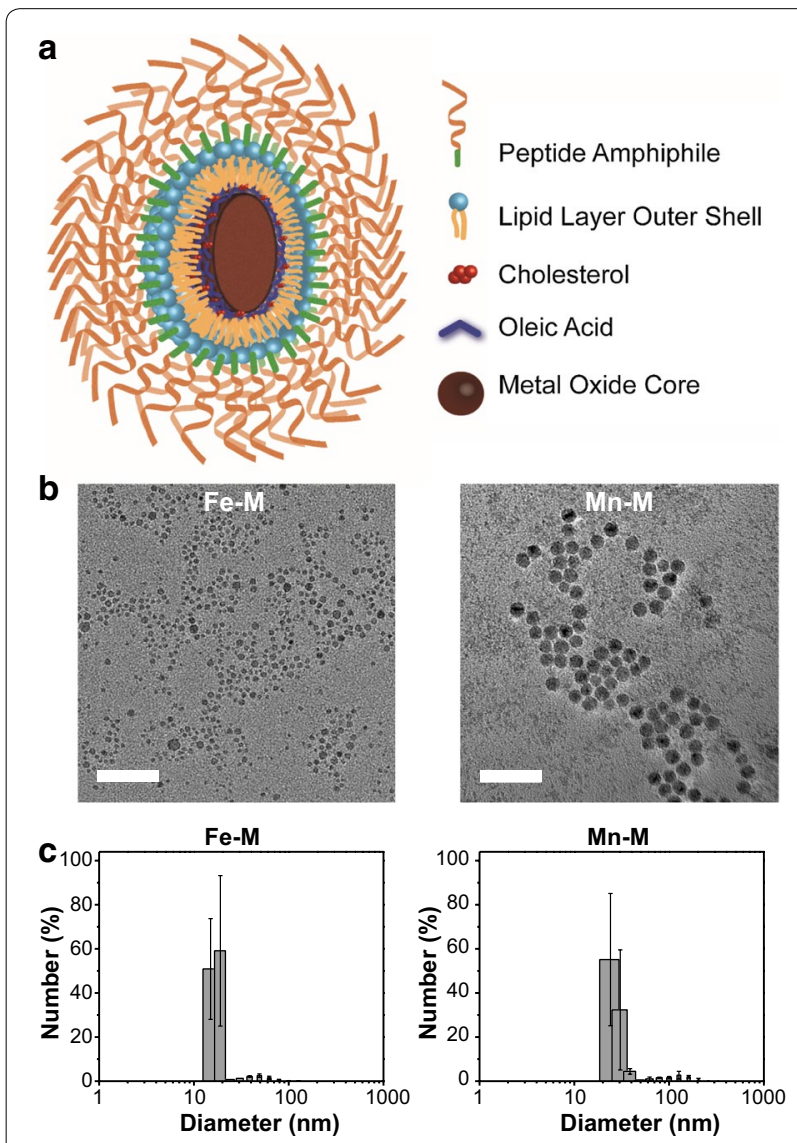

Fig. 1 Preparation and characterization of HMO-Ms. a Schematic representation of HMO-Ms. $\mathbf{b}$ TEM images of Fe-Ms and Mn-Ms after phase transfer. Scale bar $=50 \mathrm{~nm}$. c Particle size distribution of Fe-Ms and Mn-Ms determined by DLS
Table 1 Characterization of PAMs

\begin{tabular}{llll}
\hline & Diameter $(\mathbf{n m})$ & PDI & Zeta potential $(\mathbf{m V})$ \\
\hline Fe-Ms & $20.4 \pm 7.9$ & $0.151 \pm 0.063$ & $-23.8 \pm 2.3$ \\
NT-Fe-Ms & $19.8 \pm 2.4$ & $0.112 \pm 0.016$ & $-35.5 \pm 0.7$ \\
Mn-Ms & $33.1 \pm 5.5$ & $0.120 \pm 0.056$ & $-22.9 \pm 0.5$ \\
NT-Mn-Ms & $38.0 \pm 9.4$ & $0.224 \pm 0.096$ & $-26.7 \pm 6.3$ \\
\hline
\end{tabular}

and $33.1 \pm 5.5 \mathrm{~nm}$, respectively, and was similar to particles encapsulated with DSPE-PEG(2000)-CREKA only (Fig. 1b, c, Table 1 and Additional file 1: Table S3).

In order to evaluate the stability of HMO-Ms, the nanoparticles were exposed to BSA in PBS at $37{ }^{\circ} \mathrm{C}$ for $12 \mathrm{~h}$ (Additional file 1: Fig. S5). BSA bound to nanoparticles after $5 \mathrm{~min}$, increasing the particle size to $65.9 \pm 7.0 \mathrm{~nm}$ and $54.6 \pm 13.9 \mathrm{~nm}$ for $\mathrm{Fe}-\mathrm{M}$ and $\mathrm{Mn}-\mathrm{M}$, respectively. After initial BSA-binding, the particle size for both Fe-M and $\mathrm{Mn}-\mathrm{M}$ remained stable throughout the $12 \mathrm{~h}$ incubation. In contrast, HMO-Ms without the inclusion of DOPC and cholesterol in the bilayer steadily increased in particle size between 2 and $4 \mathrm{~h}$, indicating that DOPC and cholesterol is necessary to protect the nanoparticle against protein binding and aggregation.

The zeta potentials of $\mathrm{Fe}-\mathrm{Ms}$ and $\mathrm{Mn}$-Ms were - $23.8 \pm 2.3 \mathrm{mV}$ and $-22.9 \pm 0.5 \mathrm{mV}$, respectively (Table 1). The positive increase in zeta potentials of $\mathrm{Fe}-\mathrm{Ms}$ and Mn-Ms could be attributed to the presence of positively-charged arginine and lysine residues in the fibrin-binding peptide on the surface of the nanoparticles. Non-targeted HMO-Ms (NT-FeMs and NT-Mn-Ms) were similarly synthesized using DSPE-PEG(2000)-methoxy, which served as a control for in vitro studies (Additional file 1: Table S4, Figs. S6, S7). NT-Fe-Ms and NT-Mn-Ms showed particle sizes of $19.8 \pm 2.4 \mathrm{~nm}$ and $38.0 \pm 9.4 \mathrm{~nm}$, respectively, and a zeta potential of $-35.5 \pm 0.7 \mathrm{mV}$ and $-26.7 \pm 6.3 \mathrm{mV}$, respectively.

\section{In vitro release profiles}

In vitro release rates of $\mathrm{Fe}$ and $\mathrm{Mn}$ ions from HMO-Ms were investigated in PBS buffer at $37^{\circ} \mathrm{C}$ in $\mathrm{pH} 7.4$ to compare the stability between the bare metal oxide nanoparticles and HMO-Ms. The release kinetics of metal ions from HMO-Ms were much slower than that of the bare nanoparticle, thereby confirming the successful encapsulation of the metal oxide core within HMO-Ms (Fig. 2). Fe and Mn ion release was $24 \%$ and $10 \%$ for Fe-Ms and Mn-Ms, respectively, after $168 \mathrm{~h}$, while the bare Fe-NP and Mn-NP particles showed rapid burst release, with $75 \%$ total Fe and $66 \%$ total Mn release before $72 \mathrm{~h}$, thus demonstrating protection against degradation by the asymmetric bilayer. 

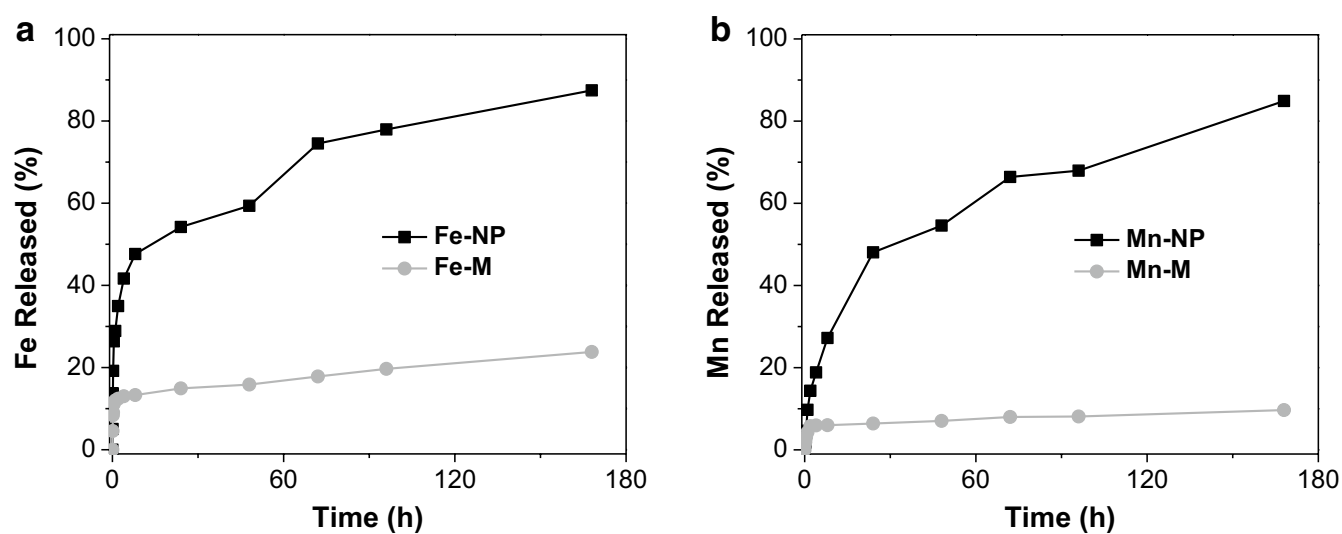

Fig. 2 In vitro release profile of Fe and Mn from bare metal oxide nanoparticles and HMO-Ms show successful encapsulation of HMO-Ms. a Fe release profiles of Fe-Ms and $\mathbf{b} \mathrm{Mn}$ release profiles of Mn-Ms in PBS buffer at $37^{\circ} \mathrm{C}$

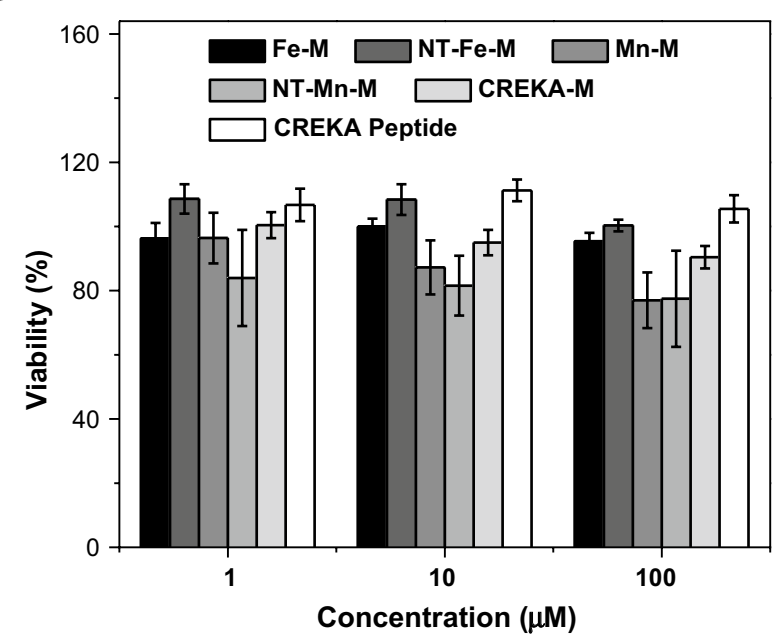

Fig. 3 HMO-Ms are biocompatible in vitro. Viability of hAECs treated with Fe-Ms, NT-Fe-Ms, Mn-Ms, NT-Mn-Ms, CREKA-Ms, and CREKA peptides at varying CREKA concentrations for $72 \mathrm{~h}$ through an MTS assay $(n=6)$

\section{In vitro biocompatibility}

Since endothelial cell activation and dysfunction are essential for the formation of atherosclerotic plaque, in vitro biocompatibility assays of HMO-Ms were carried out against human aortic endothelial cells (hAECs) (Fig. 3) [43, 44]. Upon incubation of hAECs with micelle concentrations of 1,10 , and $100 \mu \mathrm{M}$ (or 4.6, 46, and $460 \mu \mathrm{M}$ Fe or 0.92 , 9.2, and $92 \mathrm{mM} \mathrm{Mn}$ ) for $72 \mathrm{~h}$, MTS assays showed cells were over $90 \%$ viable for $\mathrm{Fe}-\mathrm{Ms}$, NT-Fe-Ms, CREKA-Ms, and free CREKA peptides as compared to the PBS control. No difference in viability was observed between Fe-Ms and CREKA-Ms, suggesting that the inclusion of the Fe-NP core to PAMs did not produce toxicity to hAECs. However, slight toxicity ( $>75 \%$ viability) was seen with the inclusion of the Mn-NP core (Mn-M and NT-Mn-M) at $100 \mu \mathrm{M}$ PAM concentration due to the cellular toxicity of $\mathrm{Mn}$ in ionic form $[25,45]$. These results indicate that Fe-Ms are more biocompatible than $\mathrm{Mn}-\mathrm{Ms}$, while $\mathrm{Mn}-\mathrm{Ms}$ were found to be non-toxic (>90\% viability) up to $10 \mu \mathrm{M}$.

\section{In vitro binding assay}

As microthrombi and fibrin deposition are found on necrotic cores of plaque, the fibrin-targeting capability of HMO-Ms was determined by an in vitro clot-binding assay (Fig. 4). Following polymerization of human plasma and thrombin, the clot was incubated with PBS, HMOMs, or NT-HMO-Ms for $1 \mathrm{~h}$ or $3 \mathrm{~h}$ at $37^{\circ} \mathrm{C}$ and evaluated for elemental analysis and imaging (Fig. 4a, b). Based on Fe content bound to the clot, Fe-Ms were found to have a 4.7-fold and 2.7-fold higher binding than NT-Fe-Ms at $1 \mathrm{~h}$ and $3 \mathrm{~h}$, respectively. Notably, Fe-Ms continued to bind over time and showed a 2.2-fold increase in binding at $3 \mathrm{~h}$ compared to $1 \mathrm{~h}$. When the CREKA targeting peptide was reduced by $50 \%$ on Fe-Ms (Fe-M-50), these nanoparticles had 2.3-fold less binding to human clots at $1 \mathrm{~h}$ incubation and 1.9-fold less binding at $3 \mathrm{~h}$ incubation than that of Fe-Ms. Similarly, Mn-Ms were found to have a 1.8-fold and 1.4-fold higher binding than its nontargeted counterpart at $1 \mathrm{~h}$ and $3 \mathrm{~h}$, respectively, which demonstrates the targeting potency and specificity of the CREKA peptide.

To visualize the binding of HMO-Ms on fibrin clots, $\mathrm{HMO}-\mathrm{Ms}$ were labeled with $\mathrm{Cy} 5$ and evaluated using confocal microscopy (Fig. 4c, d). A strong Cy5 fluorescence signal (red) of Fe-Ms was found on the fibrin clot compared to a weaker Cy5 signal observed for 

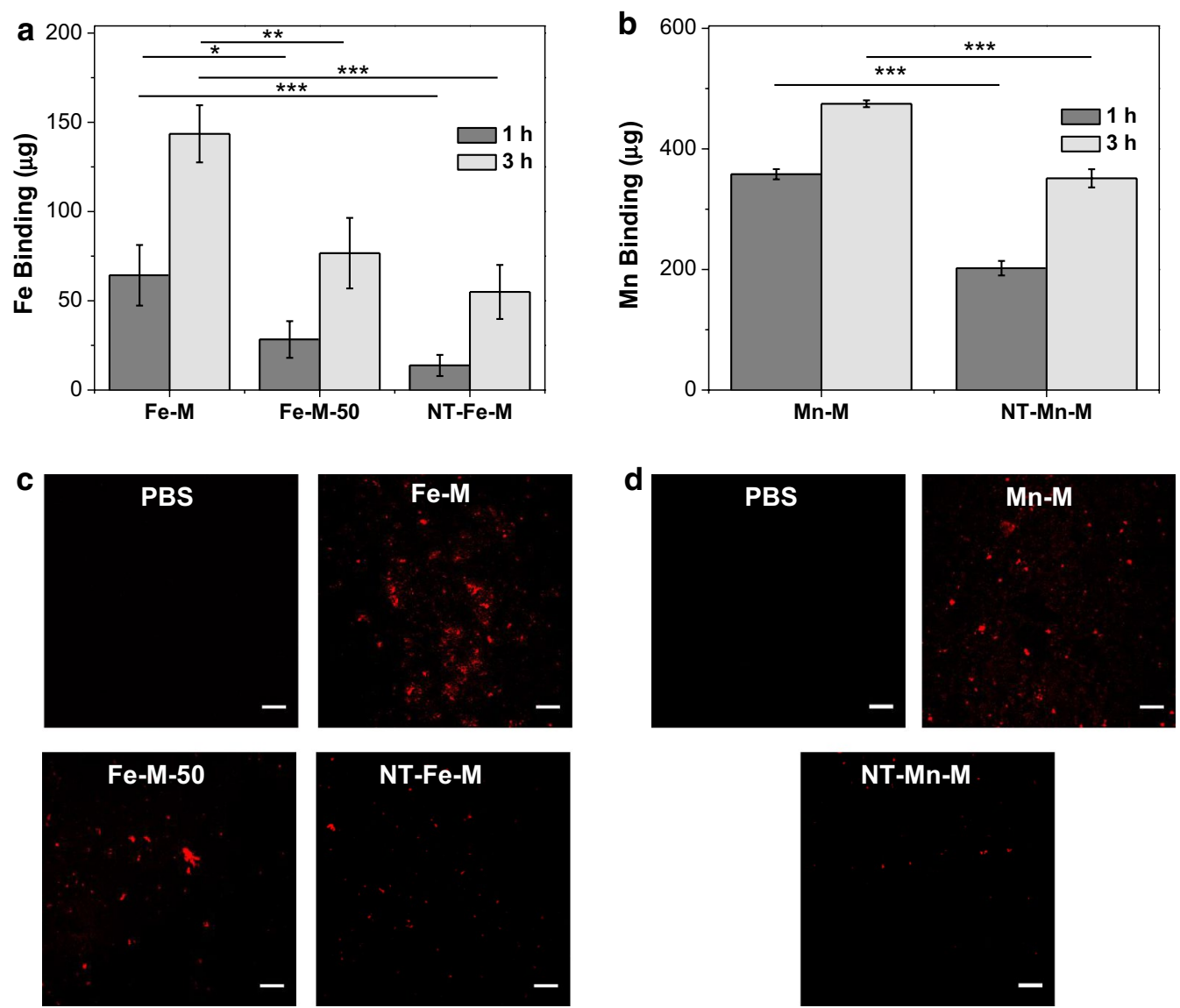

Fig. 4 HMO-Ms demonstrate higher fibrin clot binding affinity. a Fe content of fibrin clots after $1 \mathrm{~h}$ and $3 \mathrm{~h}$ exposure to Fe-Ms. b Mn content of fibrin clots after $1 \mathrm{~h}$ and $3 \mathrm{~h}$ exposure to Mn-Ms $\left(n=4 ;{ }^{*} p<0.05,{ }^{* *} p<0.01,{ }^{* * *} p<0.001\right)$. c Confocal microscopy images of Fe-Ms and $\mathbf{d}$ Cy5-Mn-Ms (red fluorescence) after $3 \mathrm{~h}$ of incubation with fibrin clots. Scale bar $=20 \mu \mathrm{m}$

Fe-Ms-50, and minimal fluorescence was found for NTFe-Ms. Similarly, Mn-Ms demonstrated the same trend in which binding affinity was higher for Mn-Ms than for NT-Mn-Ms.

\section{$r_{1}$ and $r_{2}$ measurements of HMO-Ms}

To characterize and quantify the MRI potential of HMO-Ms, MR relaxivities were determined using a 11.4 T NMR at varying concentrations of Fe-Ms and Mn-Ms (Fig. 5). The $T_{2}$-weighted Fe-Ms were found to have a longitudinal $\left(r_{1}\right)$ and transverse relaxivities $\left(r_{2}\right)$ of $0.170 \mathrm{mM}^{-1} \mathrm{~s}^{-1}$ and $456.5 \mathrm{mM}^{-1} \mathrm{~s}^{-1}$, respectively (Fig. 5a). Notably, the $r_{2}$ value from these Fe-Ms exceeded that of other reported and commercially available Fe nanoparticles in the literature $\left(50-300 \mathrm{mM}^{-1} \mathrm{~s}^{-1}\right.$ ) (Table 2). Hence, the addition of CREKA PAs and the lipid bilayer did not alter the influence of the Fe centers on the surrounding water molecules. Additionally, Mn-Ms showed enhancement in longitudinal relaxivities $\left(T_{1}\right)$. Mn-Ms exhibited an $r_{1}$ value of $0.479 \mathrm{mM}^{-1} \mathrm{~s}^{-1}$ and an $r_{2}$ value of $60.3 \mathrm{mM}^{-1} \mathrm{~s}^{-1}$, which are comparable to other Mn-NPs (Fig. 5b, Table 3).

\section{MRI using HMO-Ms on human clots}

To validate the feasibility of HMO-Ms for MRI applications, the targeting capabilities of the nanoparticles on clots were tested through MR imaging (Fig. 6). Consistent with the $r_{1}$ and $r_{2}$ values, an enhancement in signal brightness (increase for $T_{1}$ and decrease for $T_{2}$ ) was determined for Fe-Ms- and Mn-Ms-bound clots, and was statistically significant compared to non-targeted nanostructures. Both Mn-Ms and Fe-Ms were able to modify the signal intensity of MR images in a similar manner: $62 \%$ for Mn-Ms and 65\% for Fe-Ms versus 34\% and 35\% for NT-Mn-Ms and NT-Fe-Ms, respectively. The close performance of the HMO-Ms in MRI highlights the versatility of the methodology developed in this work. These results confirmed the ability of Fe-Ms and Mn-Ms to act as effective $T_{1}$ and $T_{2}$-weighted contrast agents, and both 

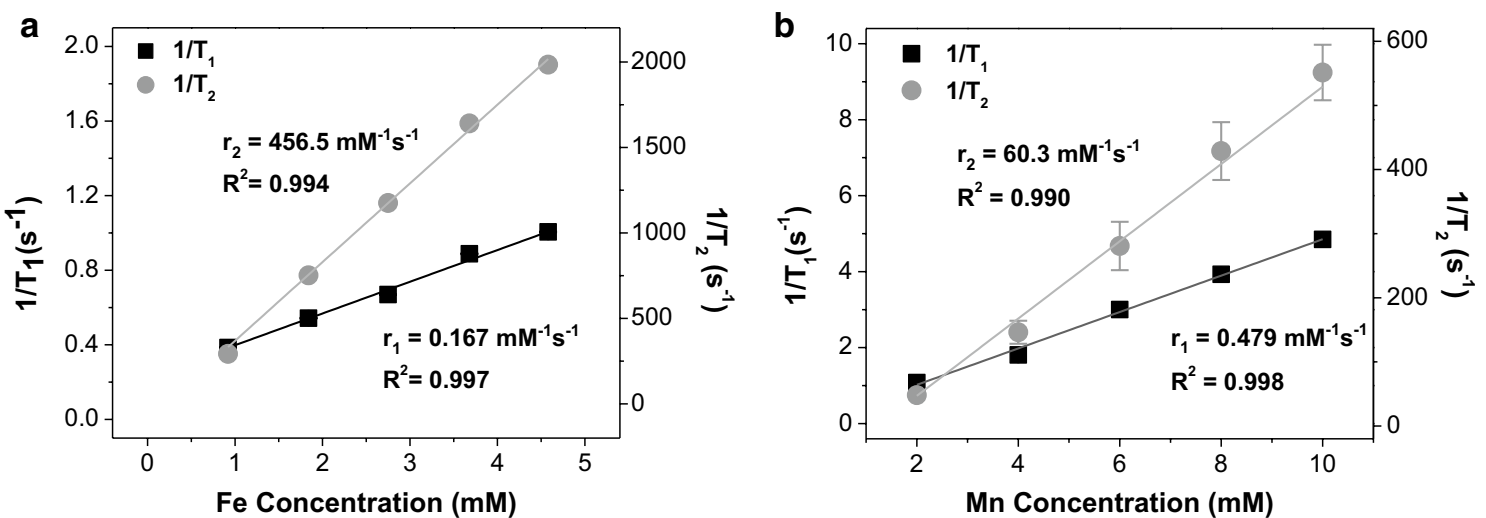

Fig. 5 HMO-Ms exhibit enhanced MR relaxivities. Longitudinal $\left(r_{1}\right.$, black) and transverse ( $r_{2}$, gray) MR relaxivity plots of a Fe-M and $\mathbf{b}$ Mn-M

Table 2 Summary of MR relaxivities of iron oxide nanoparticles in the literature

\begin{tabular}{|c|c|c|c|c|c|}
\hline Iron oxide nanoparticle & Diameter (nm) & $r_{1}\left(\mathrm{mM}^{-1} \mathrm{~s}^{-1}\right)$ & $r_{2}\left(\mathrm{mM}^{-1} \mathrm{~s}^{-1}\right)$ & Field $(\mathrm{T})$ & References \\
\hline MION & 16 & 25 & 150 & 0.47 & [47] \\
\hline PVP-grafted MNP & 14 & 2.6 & 72 & 1.5 & [48] \\
\hline USPION & 20 & 5.1 & 94 & 1.4 & [49] \\
\hline LSPIO & 39 & 11 & 119 & 9.4 & {$[20]$} \\
\hline USPION & 11 & 7.4 & 218 & 7 & {$[50]$} \\
\hline USPION & 35 & 6.6 & 128 & 3 & [51] \\
\hline USPION & 35 & 15.5 & 100 & 1.5 & [51] \\
\hline Ferumoxtran-10 & 18 & 24 & 53 & 0.47 & {$[52]$} \\
\hline Feridex & $120-180$ & 23.9 & 98.3 & 0.47 & [53] \\
\hline Resovist & 60 & 11 & 130 & 1.4 & [54] \\
\hline Feraheme & $20-30$ & 3.1 & 68 & 7 & {$[55]$} \\
\hline VSOP & 4 & 15 & 32 & 1.5 & {$[55]$} \\
\hline ZES-SPION & 4.4 & 1.5 & 17 & 7 & {$[55]$} \\
\hline $\mathrm{Fe}-\mathrm{M}$ & 20.4 & 0.2 & 457 & 11.4 & \\
\hline
\end{tabular}

MION monocrystalline iron oxide nanoparticle, $P V P$-grafted MNP poly( $N$-vinyl pyrrolidone) functionalized magnetite nanoparticles, USNP ultrasmall superparamagnetic iron oxide nanoparticle, LSPIO lipid-coated ultrasmall superparamagnetic iron particles, VSOP very small iron oxide nanoparticle, ZES-SPION zwitterion-coated exceedingly small iron oxide nanoparticle

platforms have the potential to provide important diagnostic information in MR imaging of fibrin-containing diseases like atherosclerosis.

\section{Conclusions}

In this study, we developed a hybrid nanoparticle platform that consists of a highly crystalline inorganic metal oxide core encapsulated within organic, fibrin-targeting PAs for enhanced binding to clots for applications in atherosclerosis. These hybrid nanoparticles were selfassembled and found to be monodispersed and spherical in shape with a diameter of $\sim 20-30 \mathrm{~nm}$. The DOPC and cholesterol of the asymmetric, exterior lipid bilayer of HMO-Ms provided additional stability as seen in the lack of particle aggregation upon serum incubation. In vitro, HMO-Ms were proven to be both non-toxic and biocompatible with hAECs. Moreover, HMO-Ms were found to be effective MRI contrast agents with high $r_{1}$ and $r_{2}$ relaxivities for $\mathrm{Mn}-\mathrm{M}$ and $\mathrm{Fe}-\mathrm{M}$, respectively, and upon incubation with fibrin-containing clots, the CREKA targeting moiety provided enhanced clot specificity, with three to five times higher binding than its non-targeted particles for both Mn-Ms and Fe-Ms. Our hybrid nanoplatform offers potential for early diagnosis of thrombosis in atherosclerosis, and future studies will focus on the in vivo MRI capability in mice and large animal models carrying atherosclerotic plaques.

\section{Experimental methods \\ General experimental}

All starting materials were purchased from SigmaAldrich (St. Louis, MO, USA) and Fisher (Hampton, NH, 
Table 3 Summary of MR relaxivities of manganese oxide nanoparticles in the literature

\begin{tabular}{|c|c|c|c|c|c|}
\hline $\begin{array}{l}\text { Manganese oxide } \\
\text { nanoparticle }\end{array}$ & Diameter (nm) & $r_{1}\left(m^{-1} s^{-1}\right)$ & $r_{2}\left(m^{-1} s^{-1}\right)$ & Field $(\mathrm{T})$ & References \\
\hline $\mathrm{MnO}$ & 7 & 0.37 & 1.74 & 3.0 & [38] \\
\hline $\mathrm{MnO}$ & 15 & 0.18 & 0.57 & 3.0 & [38] \\
\hline $\mathrm{MnO}$ & 20 & 0.13 & 0.52 & 3.0 & {$[38]$} \\
\hline $\mathrm{MnO}$ & 25 & 0.12 & 0.44 & 3.0 & {$[38]$} \\
\hline $\mathrm{MnO}$ & 10 & 0.81 & - & 3.0 & {$[56]$} \\
\hline $\mathrm{Mn}_{3} \mathrm{O}_{4}$ & 10 & 1.31 & 6.42 & 3.0 & {$[57]$} \\
\hline $\mathrm{Mn}_{3} \mathrm{O}_{4}$ hollow & 20 & 1.42 & 7.74 & 1.5 & [58] \\
\hline MnO hollow & 20 & 1.15 & 6.73 & 1.5 & [59] \\
\hline$M n-M$ & 33 & 0.48 & 60.3 & 11.4 & \\
\hline
\end{tabular}

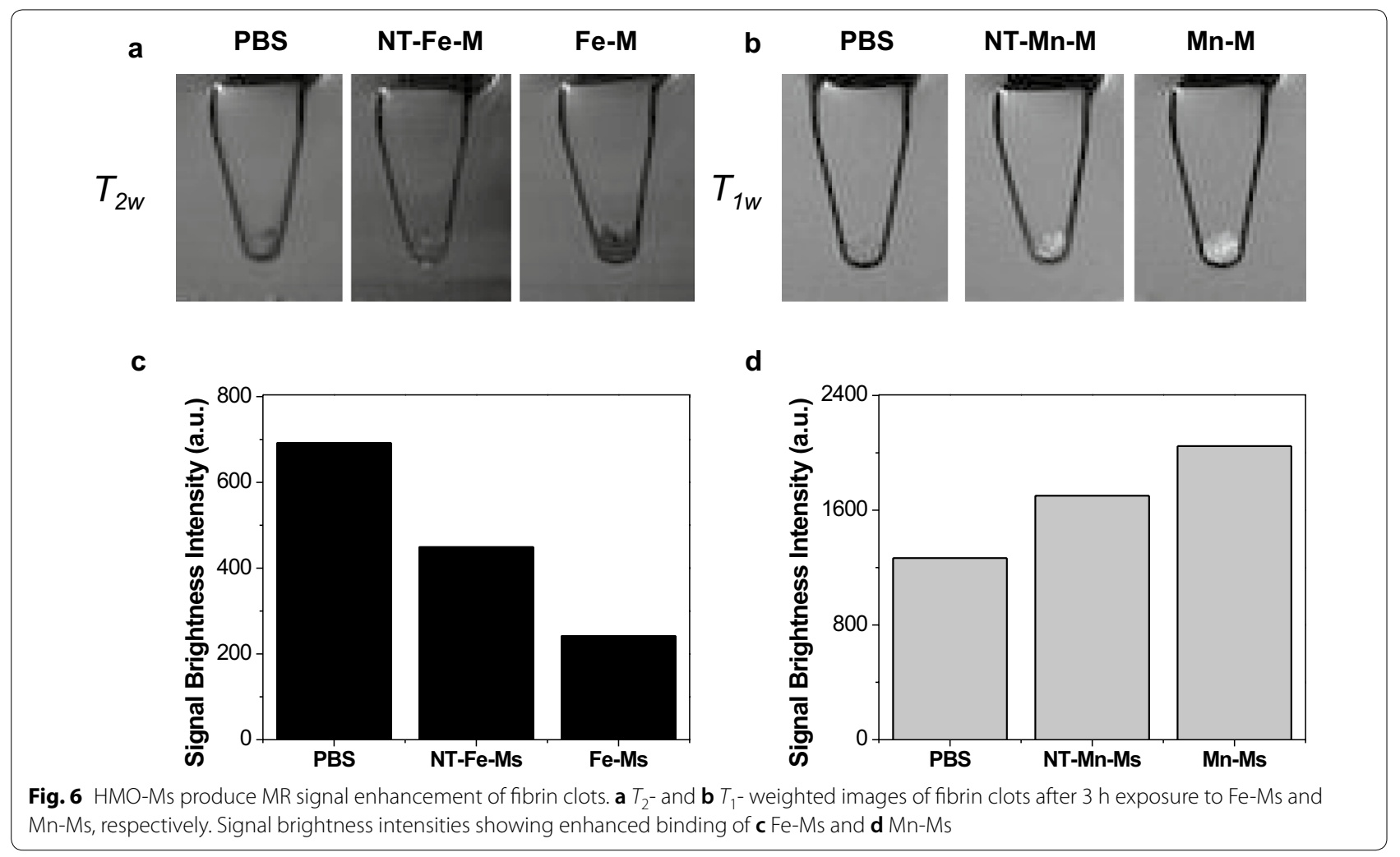

USA), unless otherwise noted, and used without further purification. 1,2-distearoyl-sn-glycero-3-phosphoethanolamine- $N$-[amino(polyethylene glycol)2000] (DSPEPEG(2000)) was purchased from Avanti Polar Lipids (USA). Human aortic endothelial cells (hAECs) were purchased from Lonza (Switzerland) and cultured in EBM-2 medium supplemented with $2 \%$ FBS and VEGF. All cell types were cultured in a humidified incubator at $37{ }^{\circ} \mathrm{C}$ under $5 \% \mathrm{CO}_{2}$. Cells at passage five were used and media was changed every 2-3 days.

\section{Preparation of iron oxide nanoparticles (Fe-NPs)}

A thermal decomposition method was followed to synthesize $\mathrm{Fe}_{3} \mathrm{O}_{4}$ nanoparticles. Briefly, oleic acid (6 mmol), oleylamine (6 mmol), 1,2-hexadecanediol (10 mmol) and $\mathrm{Fe}(\mathrm{acac})_{3}(2 \mathrm{mmol})$ were mixed into $20 \mathrm{~mL}$ of phenyl ether. The solution was heated under vigorous magnetic stirring, with reflux and Ar first at $200{ }^{\circ} \mathrm{C}$ for $30 \mathrm{~min}$, and then for another $30 \mathrm{~min}$ at $265^{\circ} \mathrm{C}$. The resulting solution had a dark brown color. After cooling down to room temperature, $40 \mathrm{~mL}$ of ethanol were added into the solution 
to precipitate the magnetic nanoparticles. The nanoparticles were separated magnetically, washed 3 times with ethanol, and then resuspended in $75 \mathrm{mM}$ oleic acid and $75 \mathrm{mM}$ oleylamine solution of hexane until further use.

\section{Preparation of manganese oxide nanoparticles (Mn-NPs)}

A two-steps protocol was followed for the preparation of manganese oxide nanoparticles. The first step was the synthesis of the manganese precursor: Sodium oleate (24.36 g) was mixed with manganese (II) chloride (7.92 g) in a solvent mixture 3:7:4 of ethanol:hexane:water $\left(140 \mathrm{~mL}\right.$ in total). This mixture was stirred at $70{ }^{\circ} \mathrm{C}$ overnight and was transferred to a separating funnel the next morning. The aqueous layer was discarded and the organic layer was washed three times with water. Finally, the organic phases were combined and evaporated to dryness to give a pink-red solid product of manganese oleate. To prepare $\mathrm{MnO}$ nanoparticles, manganese oleate $(1.24 \mathrm{~g})$ was dissolved in 1-hexadecene $(10 \mathrm{~g})$ in a 3-neck round-bottom flask with a reflux system. The solution was heated to $80{ }^{\circ} \mathrm{C}$ under vacuum to remove hexanewater. After 90 min, the vacuum was exchanged for $\mathrm{N}_{2}$ and the temperature was raised to $280^{\circ} \mathrm{C}$ and maintained for $30 \mathrm{~min}$. The dark solution was allowed to cool down to room temperature and acetone $(10 \mathrm{~mL})$ was added to precipitate the nanoparticles. The particles were then centrifuged and washed again with acetone. Finally, they were resuspended in $75 \mathrm{mM}$ oleic acid hexane solution and stored in the fridge until further use.

\section{Synthesis of CREKA peptide}

CREKA peptide was synthesized according to protocols in the literature [36]. Briefly, the peptide was synthesized on a Rink resin from $\mathrm{C}$-terminus to $\mathrm{N}$-terminus via the Fmoc/t-Bu strategy on an automated peptide synthesizer (PS3, Protein Technologies, Tucson, AZ, USA). Cysteine was added on the $\mathrm{N}$-terminus for conjugation to DSPEPEG(2000)-maleimide. A solution of 94:2:5:2:5:1 vol\% TFA:1,2-ethanedithiol:water:triisopropylsilane was used to cleave the peptide off the resin. The resulting cleaved peptide was precipitated and washed twice with ice-cold diethyl ether. The crude peptide was purified by reversephase high performance liquid chromatography (HPLC) on a Luna C8 column $(250 \times 10 \mathrm{~mm}$ ID, $5 \mathrm{~mm}$, Phenomenex, Torrance, CA, USA) using at $55{ }^{\circ} \mathrm{C}$ using $0.1 \%$ formic acid in acetonitrile/water mixtures and characterized by matrix-assisted laser desorption/ionization (MALDI) mass spectroscopy.

\section{Synthesis of DSPE-PEG(2000)-CREKA}

The cysteine containing peptide was conjugated onto DSPE-PEG(2000)-maleimide to afford
DSPE-PEG-peptide conjugate via thioether linkage. After $24 \mathrm{~h}$ at room temperature, the crude product was purified by HPLC and characterized by MALDI as described above. A fluorescent-labeled Cy5 DSPE-PEG(2000) conjugate was also synthesized using similar methods.

\section{Encapsulation of hybrid metal oxide peptide amphiphile micelles (HMO-Ms)}

HMO-Ms were self-assembled by adding a chloroform solution of DOPC, cholesterol, and DSPE-PEG(2000)CREKA $\quad$ (DOPC: cholesterol:amphiphiles $=1.33: 1.33$ :1 molar ratio) with Fe-NP or Mn-NP. The chloroform solution was stirred and evaporated under nitrogen. The resulting film was dried overnight and hydrated at $80^{\circ} \mathrm{C}$ for $30 \mathrm{~min}$ in water or phosphate buffered saline (PBS), and the HMO-M solution was allowed to cool to room temperature.

\section{Characterization of bare metal oxide nanoparticles and HMO-Ms}

Bare metal oxide nanoparticles were imaged through high resolution transmission electron microscopy (HRTEM, JEM-2100F, JEOL, Tokyo, Japan) at a working voltage of $200 \mathrm{kV}$. Energy-dispersive X-ray spectroscopy (EDXS, JEM-2100F, JEOL, Tokyo, Japan) was utilized to analyze the Fe and Mn concentration of Fe-NPs and MnNPs, respectively. The bare Fe-NP samples were diluted (1:1000) and applied onto a 400 mesh carbon-coated $\mathrm{Cu}$ grid (Agar Scientific, UK) followed by evaporation of solvent under vacuum overnight. Particle sizes and zeta potentials of $100 \mu \mathrm{M}$ HMO-Ms were analyzed using DLS (Mobius, Wyatt, Santa Barbara, CA, USA). All DLS and zeta potential measurements were performed in three replicas and reported as mean \pm standard deviation. To visualize the morphology of the nanoparticles, $100 \mu \mathrm{M}$ PAM solutions were negatively stained with 2 wt\% uranyl acetate solution on 400 mesh carbon grids (Ted Pella, Redding, CA, USA) and imaged using TEM (JEM-2100F, JEOL, Tokyo, Japan).

\section{Stability studies of HMO-Ms with and without DOPC and cholesterol}

$100 \mu \mathrm{M}$ of Fe-Ms and Mn-Ms with and without DOPC and cholesterol were incubated with $5 \mathrm{mg} / \mathrm{mL}$ bovine serum albumin (BSA) at $37{ }^{\circ} \mathrm{C}$ in PBS. A baseline particle size measurement was detected using DLS prior to the addition of BSA. Particle size and distribution were measured every $2 \mathrm{~h}$ for $12 \mathrm{~h}$ to determine the particle stability in suspension over time. 


\section{Release profiles of HMO-Ms}

Elemental release of $\mathrm{Fe}$ and $\mathrm{Mn}$ from bare metal oxide nanoparticles and $\mathrm{HMO}-\mathrm{Ms}$ were performed in $250 \mathrm{~mL}$ in PBS buffer at $37^{\circ} \mathrm{C}$ in $\mathrm{pH}$ 7.4. In a 10,000 MWCO pleated dialysis bag (Fisher, St. Louis, MO, USA) Fe-Ms and Mn-Ms containing $0.92 \mu \mathrm{mol} \mathrm{Fe}$ or $2 \mu \mathrm{mol}$ Mn were suspended in $2 \mathrm{~mL}$ PBS. At predetermined time points, $1 \mathrm{~mL}$ aliquots were taken from the PBS solution and replenished with fresh buffer solution. The removed aliquot was digested in concentrated nitric acid and analyzed for Fe or Mn via inductively coupled plasma optical emission spectrometry (ICP-OES, iCap 7400 Analyzer, ThermoFisher).

\section{In vitro biocompatibility}

hAECs were plated in 96-well plates at a cell density of 2000 cells/well and varying concentrations of CREKA peptide, CREKA-Ms, Fe-Ms, and Mn-Ms in PBS were added to wells. The plates were incubated at $37^{\circ} \mathrm{C}$ and $5 \%$ $\mathrm{CO}_{2}$ for $72 \mathrm{~h}$, and cell viability was determined via MTS assay (BioVision, Milpitas, CA, USA) according to manufacturer's instructions. Absorbance at $490 \mathrm{~nm}$ was used to measure the enzymatic activity level, and $100 \%$ viability using non-treated, healthy cells was used as a positive control. Viability of each treatment group was measured from six replicates.

\section{Fibrin clot binding}

To evaluate the binding affinity of HMO-Ms, fibrin-containing clots were synthesized based on previous literature [46]. Briefly, human plasma $(225 \mu \mathrm{L})$ was mixed with $75 \mu \mathrm{L}$ of $100 \mathrm{mM} \mathrm{CaCl}_{2}$ solution. Thrombin (5 units at $0.5 \mathrm{unit} / \mu \mathrm{L}$ ) was added to the plasma and stirred evenly. The fibrin clot was allowed to incubate at room temperature for $30 \mathrm{~min}$ or until it turned into a gel. PBS, bare metal oxide nanoparticles, or HMO-Ms at $100 \mu \mathrm{M}$ PAM (or $457 \mu \mathrm{M}$ Fe or $920 \mu \mathrm{M} \mathrm{Mn}$ ) were added onto the fibrin clot and incubated for $1 \mathrm{~h}$ or $3 \mathrm{~h}$ at $37^{\circ} \mathrm{C}$. The clot was washed three times with PBS prior to analysis. For elemental analysis, the fibrin clot was digested with concentrated nitric acid and analyzed for Fe or Mn amount by ICP-OES. For confocal microscopy, the fibrin clot was incubated with Cy5-labeled PAMs (10 mol\% Cy5 amphiphiles) for $3 \mathrm{~h}$ and fixed with $4 \%$ paraformaldehyde, prior to observation using confocal laser scanning microscopy (CLSM, Zeiss, Oberkochen, Germany) at excitation wavelengths of $650 \mathrm{~nm}$ to visualize the nanoparticle (red).

\section{$r_{1}$ and $r_{2}$ analysis}

Using $500 \mathrm{MHz} \mathrm{NMR}$ at $37{ }^{\circ} \mathrm{C}(11.4 \mathrm{~T}$, Varian), longitude $\left(\mathrm{T}_{1}\right)$ and transverse $\left(\mathrm{T}_{2}\right)$ relaxation rate was determined at six different concentration levels $(0-4.6 \mathrm{mM} \mathrm{Fe}$ or $0-1000 \mu \mathrm{M}$ PAMs and $0-10 \mathrm{mM}$ Mn or $0-1000 \mu \mathrm{M}$ PAMs). All relaxivity values were obtained as the sloped associated with the linear fit of Fe and Mn concentration, respectively, v. $1 / T_{1}$ or $1 / T_{2}$.

\section{MRI analysis on fibrin clot}

For MR imaging the clots were transferred to PCR microtubes, centrifuged for $60 \mathrm{~s}$ at $10,000 \mathrm{rpm}$ to 'pellet' the clots and then $200 \mu \mathrm{L}$ of water were added into each tube. The microtubes were then mounted on a custom printed poly-(lactic acid) holder and imaged in a 3.0 T horizontal bore MR Solutions Benchtop MRI system equipped with $48 \mathrm{G} / \mathrm{cm}$ actively shielded gradients. To image the samples, a $56-\mathrm{mm}$ diameter quadrature birdcage coil was used in transmit/receive mode. All MR images were acquired with an image matrix $256 \times 252$, FOV $60 \times 60 \mathrm{~mm}, 2$ sets of 3 slices each with a slice thickness of $1 \mathrm{~mm}$. For $T_{2}$-weighted imaging, fast spin echo (FSE) sequences with the following parameters were used: $\mathrm{T}_{E}=68 \mathrm{~ms}, \mathrm{~T}_{\mathrm{R}}=4800 \mathrm{~ms}, \mathrm{~N}_{\mathrm{A}}=10, \mathrm{~A}_{\mathrm{T}}=24 \mathrm{~m} 53 \mathrm{~s}$. For $T_{1}$-weighted imaging, fast spin echo (FSE) sequences with the following parameters were used: $T_{E}=11 \mathrm{~ms}$, $\mathrm{T}_{\mathrm{R}}=720 \mathrm{~ms}, \mathrm{~N}_{\mathrm{A}}=20, \mathrm{~A}_{\mathrm{T}}=15 \mathrm{~m} 41 \mathrm{~s}$.

\section{Statistical analysis}

For all experiments in this work, mean $\pm \mathrm{SD}$ was calculated. A two-tailed Student $t$-tests and a one-way analysis of variance (ANOVA) was used to determine statistical significance between two groups and more than two groups, respectively. A $P$ value $<0.05$ was considered statistically significant.

\section{Additional file}

Additional file 1. Additional figures and tables.

Abbreviations

MRI: magnetic resonance imaging; HMO-Ms: hybrid metal oxide peptide amphiphile micelles; Fe-Ms: iron oxide peptide amphiphile micelles; MnMs: manganese oxide peptide amphiphile micelles; hAECs: human aortic endothelial cells; CVD: cardiovascular disease; EPR: enhanced permeability and retention; Fe-NPs: iron oxide nanoparticles; Mn-NPs: manganese oxide nanoparticles; PAMs: peptide amphiphile micelles; TEM: transmission electron microscopy; PAs: peptide amphiphiles; DLS: dynamic light scattering; DOPC: 1,2-dioleoyl-sn-glycero-3-phosphocholine; NT-HMO-Ms: non-targeted HMO-Ms.

\section{Authors' contributions}

$C P, M B L$ and EJC conceived the project and designed the experiments; $C P, J G$, $J J, T C, M B L$, and EJC performed the experiments and analyzed the data; CP, $M B L$ and EJC wrote and edited the manuscript. All authors read and approved the final manuscript. 


\begin{abstract}
Author details
1 Department of Biomedical Engineering, University of Southern California, 1042 Downey Way, Los Angeles, CA 90089, USA. ${ }^{2}$ Advanced (Magnetic) Theranostic Nanostructures Lab, Department of Life Sciences, International Iberian Nanotechnology Laboratory, Avenida Mestre José Veiga, Braga, Portugal. ${ }^{3}$ Department of Materials Science and Chemical Engineering, University of Southern California, 925 Bloom Walk, Los Angeles, CA 90089, USA. ${ }^{4}$ Eli and Edythe Broad Center for Regenerative Medicine and Stem Cell Research, Keck School of Medicine, University of Southern California, Los Angeles, CA, USA. ${ }^{5}$ Division of Nephrology and Hypertension, Department of Medicine, Keck School of Medicine, University of Southern California, Los Angeles, CA, USA. ${ }^{6}$ Norris Comprehensive Cancer Center, Keck School of Medicine, University of Southern California, Los Angeles, CA, USA.
\end{abstract}

\section{Acknowledgements}

The authors thank Mr. Allan Kershaw, Miss Zhangjingyi Jiang, and Mr. Victor Ong for experimental assistance. The authors thank the Center for Electron Microscopy and Microanalysis, Center of Excellence in NanoBiophysics, Center of Excellence for Molecular Characterization, and Translational Imaging Center at the University of Southern California for assistance in instrumental setups.

\section{Competing interests}

The authors declare that they have no competing interests.

\section{Availability of data and materials}

The datasets used and/or analyzed during the current study are available from the corresponding author on reasonable request.

\section{Consent for publication}

All authors have provided consent for the manuscript to be published.

\section{Ethics approval and consent to participate}

Not applicable.

\section{Funding}

This work was support by the University of Southern California, the National Heart, Lung, and Blood Institute (NHLBI, ROOHL124279), Eli and Edythe Broad Innovation Award, and the L.K. Whittier Foundation Non-Cancer Translational Research Award granted to E.J.C. M.B.L. and J.G. acknowledge the support from NORTE 2020 (2014-2020 North Portugal Regional Operational Programme) and the ERDF (European Regional Development Fund) under the Grant NORTE-45-2015-02, and by European Union's Horizon 2020 research and innovation program under Grant Agreement N ${ }^{\circ} 686009$.

\section{Publisher's Note}

Springer Nature remains neutral with regard to jurisdictional claims in published maps and institutional affiliations.

Received: 11 August 2018 Accepted: 7 November 2018 Published online: 15 November 2018

\section{References}

1. Mozaffarian D, Benjamin EJ, Go AS, Arnett DK, Blaha MJ, Cushman M, Das SR, de Ferranti S, Despres J-P, Fullerton HJ, et al. Heart disease and stroke statistics-2016 update: a report from the American Heart Association. Circulation. 2016;133:e38-60.

2. Bourantas CV, Garcia-Garcia HM, Farooq V, Diletti R, Muramatsu T, Maehara A, Xu K, Genereux P, Fahy M, Weisz G, et al. Clinical and angiographic characteristics of patients likely to have vulnerable plaques: analysis from the PROSPECT study. JACC Cardiovasc Imaging. 2013;6:1263-72.

3. Falk E. Pathogenesis of atherosclerosis. J Am Coll Cardiol. 2006;47:C7-12.

4. Rahmani M, Cruz RP, Granville DJ, McManus BM. Allograft vasculopathy versus atherosclerosis. Circ Res. 2006;99:801-15.

5. Tarkin JM, Dweck MR, Evans NR, Takx RAP, Brown AJ, Tawakol A, Fayad ZA, Rudd JHF. Imaging atherosclerosis. Circ Res. 2016;118:750-69.

6. Karczmar GS, Fan X, Al-Hallaq HA, Zamora M, River JN, Rinker-Schaeffer C, Zaucha M, Tarlo K, Kellar K. Uptake of a superparamagnetic contrast agent imaged by MR with high spectral and spatial resolution. Magn Reson Med. 2000;43:633-9.

7. Leoni L, Roman BB. MR imaging of pancreatic islets: tracking isolation, transplantation and function. Curr Pharm Des. 2010;16:1582-94.

8. Kim Y, Lobatto ME, Kawahara T, Chung BL, Mieszawska AJ, Sanchez-Gay$\tan B$ L, Fay F, Senders ML, Calcagno C, Becraft J, et al. Probing nanoparticle translocation across the permeable endothelium in experimental atherosclerosis. Proc Natl Acad Sci USA. 2014;111:1078-83.

9. England CG, Im H-J, Feng L, Chen F, Graves SA, Hernandez R, Orbay H, Xu C, Cho SY, Nickles RJ, et al. Re-assessing the enhanced permeability and retention effect in peripheral arterial disease using radiolabeled long circulating nanoparticles. Biomaterials. 2016;100:101-9.

10. Palekar RU, Jallouk AP, Lanza GM, Pan H, Wickline SA. Molecular imaging of atherosclerosis with nanoparticle-based fluorinated MRI contrast agents. Nanomedicine. 2015;10:1817-32.

11. Yavuz MS, Cheng Y, Chen J, Cobley CM, Zhang Q, Rycenga M, Xie J, Kim C, Song $\mathrm{KH}$, Schwartz AG, et al. Gold nanocages covered by smart polymers for controlled release with near-infrared light. Nat Mater. 2009;8:935-9.

12. Lee JE, Lee N, Kim H, Kim J, Choi SH, Kim JH, Kim T, Song IC, Park SP, Moon WK, Hyeon T. Uniform mesoporous dye-doped silica nanoparticles decorated with multiple magnetite nanocrystals for simultaneous enhanced magnetic resonance imaging, fluorescence imaging, and drug delivery. J Am Chem Soc. 2010;132:552-7.

13. Sajja HK, East MP, Mao H, Wang YA, Nie S, Yang L. Development of multifunctional nanoparticles for targeted drug delivery and noninvasive imaging of therapeutic effect. Curr Drug Discov Technol. 2009;6:43-51.

14. Cheon J, Lee J-H. Synergistically integrated nanoparticles as multimodal probes for nanobiotechnology. Acc Chem Res. 2008;41:1630-40.

15. Fang J, Nakamura $H$, Maeda $H$. The EPR effect: unique features of tumor blood vessels for drug delivery, factors involved, and limitations and augmentation of the effect. Adv Drug Deliv Rev. 2011;63:136-51.

16. Bruckman MA, Yu X, Steinmetz NF. Engineering Gd-loaded nanoparticles to enhance MRI sensitivity via T1 shortening. Nanotechnology. 2013;24:462001.

17. Zavaleta C, Ho D, Chung EJ. Theranostic nanoparticles for tracking and monitoring disease state. SLAS Technol. 2018;23:281-93.

18. Dizaj SM, Jafari S, Khosroushahi AY. A sight on the current nanoparticlebased gene delivery vectors. Nanoscale Res Lett. 2014;9:1-9.

19. Zhao F, Yao D, Guo R, Deng L, Dong A, Zhang J. Composites of polymer hydrogels and nanoparticulate systems for biomedical and pharmaceutical applications. Nanomaterials. 2015;5:2054-130.

20. Briley-Saebo KC, Cho YS, Shaw PX, Ryu SK, Mani V, Dickson S, Izadmehr E, Green S, Fayad ZA, Tsimikas S. Targeted iron oxide particles for in vivo magnetic resonance detection of atherosclerotic lesions with antibodies directed to oxidation-specific epitopes. J Am Coll Cardiol. 2011;57:337-47.

21. Blanco-Andujar C, Walter A, Cotin G, Bordeianu C, Mertz D, FelderFlesch D, Begin-Colin S. Design of iron oxide-based nanoparticles for MRI and magnetic hyperthermia. Nanomedicine. 2016;11:1889-910.

22. Pan D, Caruthers SD, Senpan A, Schmieder AH, Wickline SA, Lanza GM. Revisiting an old friend: manganese-based MRI contrast agents. Wiley Interdiscip Rev Nanomed Nanobiotechnol. 2011;3:162-73.

23. Ittrich H, Peldschus K, Raabe N, Kaul M, Adam G. Superparamagnetic iron oxide nanoparticles in biomedicine: applications and developments in diagnostics and therapy. Rofo. 2013;185:1149-66.

24. Wei H, Insin N, Lee J, Han H-S, Cordero JM, Liu W, Bawendi MG. Compact zwitterion-coated iron oxide nanoparticles for biological applications. Nano Lett. 2012;12:22-5.

25. Pan D, Schmieder AH, Wickline SA, Lanza GM. Manganese-based MRI contrast agents: past, present, and future. Tetrahedron. 2011;67:8431-44.

26. Turner CT, Mclnnes SJP, Voelcker NH. Cowin AJ: Therapeutic potential of inorganic nanoparticles for the delivery of monoclonal antibodies. J Nanomater. 2015;2015:309602. https://doi.org/10.1155/2015/309602.

27. Tonga GY, Moyano DF, Kim CS, Rotello VM. Inorganic nanoparticles for therapeutic delivery: trials, tribulations and promise. Curr Opin Colloid Interface Sci. 2014;19:49-55

28. Yuan J, Xu Y, Mueller AHE. One-dimensional magnetic inorganic-organic hybrid nanomaterials. Chem Soc Rev. 2011;40:640-55. 
29. Vivero-Escoto JL, Huang Y-T. Inorganic-organic hybrid nanomaterials for therapeutic and diagnostic imaging applications. Int J Mol Sci. 2011;12:3888-927.

30. Pellegrino T, Manna L, Kudera S, Liedl T, Koktysh D, Rogach AL, Keller S, Raedler J, Natile G, Parak WJ. Hydrophobic nanocrystals coated with an amphiphilic polymer shell: a general route to water soluble nanocrystals. Nano Lett. 2004;4:703-7.

31. Tromsdorf UI, Bigall NC, Kaul MG, Bruns OT, Nikolic MS, Mollwitz B, Sperling RA, Reimer R, Hohenberg H, Parak WJ, et al. Size and surface effects on the MRI relaxivity of manganese ferrite nanoparticle contrast agents. Nano Lett. 2007;7:2422-7.

32. Peters D, Kastantin M, Kotamraju VR, Karmali PP, Gujraty K, Tirrell M, Ruoslahti E. Targeting atherosclerosis by using modular, multifunctional micelles. Proc Natl Acad Sci USA. 2009;106:9815-9.

33. Chung EJ, Pineda F, Nord K, Karczmar G, Lee S-K, Tirrell M. Fibrin-targeting, peptide amphiphile micelles as contrast agents for molecular MRI. J Cell Sci Ther. 2014;5:1000184.

34. Yoo SP, Barrett JC, Tirrell M, Chung EJ, Pineda F, Poon C. Gadoliniumfunctionalized peptide amphiphile micelles for multimodal imaging of atherosclerotic lesions. ACS Omega. 2016;1:996-1003.

35. Chung EJ, Mlinar LB, Sugimoto MJ, Nord K, Roman BB, Tirrell M. In vivo biodistribution and clearance of peptide amphiphile micelles. Nanomedicine. 2015;11:479-87.

36. Chung EJ, Cheng Y, Morshed R, Nord K, Han Y, Wegscheid ML, Auffinger B, Wainwright DA, Lesniak MS, Tirrell MV. Fibrin-binding, peptide amphiphile micelles for targeting glioblastoma. Biomaterials. 2014;35:1249-56.

37. Sun S, Zeng H, Robinson DB, Raoux S, Rice PM, Wang SX, Li G. Monodisperse $\mathrm{MFe}_{2} \mathrm{O}_{4}(\mathrm{M}=\mathrm{Fe}, \mathrm{Co}, \mathrm{Mn})$ nanoparticles. J Am Chem Soc. 2004;126:273-9.

38. Na HB, Lee JH, An K, Park YI, Park M, Lee IS, Nam D-H, Kim ST, Kim S-H, Kim S-W, et al. Development of a T1 contrast agent for magnetic resonance imaging using $\mathrm{MnO}$ nanoparticles. Angew Chem Int Ed. 2007:46:5397-401.

39. Yousefi N, Tufenkji N. Probing the interaction between nanoparticles and lipid membranes by quartz crystal microbalance with dissipation monitoring. Front Chem. 2016:4:76/71-76/78.

40. Peetla C, Stine A, Labhasetwar V. Biophysical interactions with model lipid membranes: applications in drug discovery and drug delivery. Mol Pharm. 2009;6:1264-76.

41. Weis RM, McConnell HM. Cholesterol stabilizes the crystal-liquid interface in phospholipid monolayers. J Phys Chem. 1985;89:4453-9.

42. Wennberg CL, van der Spoel D, Hub JS. Large influence of cholesterol on solute partitioning into lipid membranes. J Am Chem Soc. 2012;134:5351-61.

43. Khodabandehlou K, Masehi-Lano JJ, Poon C, Wang J, Chung EJ. Targeting cell adhesion molecules with nanoparticles using in vivo and flow-based in vitro models of atherosclerosis. Exp Biol Med. 2017;242:799-812.

44. Newby AC, Zaltsman AB. Fibrous cap formation or destruction-the critical importance of vascular smooth muscle cell proliferation, migration and matrix formation. Cardiovasc Res. 1999;41:345-60.
45. Zhen Z, Xie J. Development of manganese-based nanoparticles as contrast probes for magnetic resonance imaging. Theranostics. 2012;2:45-54.

46. Wen AM, Jiang K, Lee KL, Yang AC, Yu X, Wang Y, Gao H, Simon DI, Hsu GC, Steinmetz NF. Shaping bio-inspired nanotechnologies to target thrombosis for dual optical-magnetic resonance imaging. J Mater Chem B. 2015;3:6037-45.

47. LaConte LEW, Nitin N, Zurkiya O, Caruntu D, O'Connor CJ, Hu X, Bao G Coating thickness of magnetic iron oxide nanoparticles affects $\mathrm{R} 2$ relaxivity. J Magn Reson Imaging. 2007;26:1634-41.

48. Fattahi H, Arsalani N, Nazarpoor M. Synthesis and characterization of PVP-functionalized superparamagnetic $\mathrm{Fe}_{3} \mathrm{O}_{4}$ nanoparticles as an MRI contrast agent. Express Polym Lett. 2010;4(6):329-38.

49. Li P, Chevallier P, Ramrup P, Biswas D, Vuckovic D, Fortin M-A, Oh JK. Mussel-inspired multidentate block copolymer to stabilize ultrasmall superparamagnetic $\mathrm{Fe}_{3} \mathrm{O}_{4}$ for magnetic resonance imaging contrast enhancement and excellent colloidal stability. Chem Mater. 2015;27:7100-9.

50. Chee HL, Gan CRR, Ng M, Low L, Fernig DG, Bhakoo KK, Paramelle D. Biocompatible peptide-coated ultrasmall superparamagnetic iron oxide nanoparticles for in vivo contrast-enhanced magnetic resonance imaging. ACS Nano. 2018;12:6480-91.

51. Simon GH, Bauer J, Saborovski O, Fu Y, Corot C, Wendland MF, DaldrupLink HE. T1 and T2 relaxivity of intracellular and extracellular USPIO at 1.5T and 3T clinical MR scanning. Eur Radiol. 2006;16:738-45.

52. Vassallo P, Matei C, Heston WD, McLachlan SJ, Koutcher JA, Castellino RA. AMI-227-enhanced MR lymphography: usefulness for differentiating reactive from tumor-bearing lymph nodes. Radiology. 1994;193:501-6.

53. Wang Y-XJ. Superparamagnetic iron oxide based MRI contrast agents: current status of clinical application. Quant Imaging Med Surg. 2011;1:35-40.

54. Tromsdorf UI, Bruns OT, Salmen SC, Beisiegel U, Weller H. A highly effective, nontoxic T1 MR contrast agent based on ultrasmall PEGylated iron oxide nanoparticles. Nano Lett. 2009;9:4434-40.

55. Wei H, Bruns OT, Kaul MG, Hansen EC, Barch M, Wisniowska A, Chen O, Chen Y, Li N, Okada S, et al. Exceedingly small iron oxide nanoparticles as positive MRI contrast agents. Proc Natl Acad Sci USA. 2017;114:2325-30.

56. Lu Y, Zhang L, Li J, Su Y-D, Liu Y, Xu Y-J, Dong L, Gao H-L, Lin J, Man $\mathrm{N}$, et al. MnO nanocrystals: a platform for integration of MRI and genuine autophagy induction for chemotherapy. Adv Funct Mater. 2013;23:1534-46.

57. Huang C-C, Khu N-H, Yeh C-S. The characteristics of sub $10 \mathrm{~nm}$ manganese oxide $T 1$ contrast agents of different nanostructured morphologies. Biomaterials. 2010;31:4073-8.

58. Shin J, Anisur RM, Ko MK, Im GH, Lee JH, Lee IS. Hollow manganese oxide nanoparticles as multifunctional agents for magnetic resonance imaging and drug delivery. Angew Chem Int Ed. 2009;48:321-4.

59. Hsieh C-H, Chang M-T, Chien Y-J, Chou L-J, Chen L-J, Chen C-D. Coaxial metal-oxide-semiconductor (MOS) $\mathrm{Au} / \mathrm{Ga}_{2} \mathrm{O}_{3} / \mathrm{GaN}$ nanowires. Nano Lett. 2008;8:3288-92.

Ready to submit your research? Choose BMC and benefit from

- fast, convenient online submission

- thorough peer review by experienced researchers in your field

- rapid publication on acceptance

- support for research data, including large and complex data types

- gold Open Access which fosters wider collaboration and increased citations

- maximum visibility for your research: over $100 \mathrm{M}$ website views per year

At BMC, research is always in progress.

Learn more biomedcentral.com/submissions 